\title{
KEY FEATURES OF BEACH HANDBALL SELF-ORGANIZATION: COMPARISON BETWEEN GENDERS
}

original paper

() Wroclaw University of Health and Sport Sciences

DOI: https://doi.org/10.5114/hm.2022.107971

\author{
FELIPE MODOLO ${ }^{1}$, WALMIR ROMÁRIO DOS SANTOS ${ }^{1}$, \\ RENATO RESINA DE OLIVEIRA CAMPOS ${ }^{2}$, MÁRCIO PEREIRA MORATO ${ }^{1}$, \\ RAFAEL POMBO MENEZES ${ }^{1}$ \\ ${ }^{1}$ School of Physical Education and Sport of Ribeirão Preto, University of São Paulo, Ribeirão Preto, Brazil \\ ${ }^{2}$ Faculty of Human Kinetics, University of Lisbon, Lisbon, Portugal
}

\begin{abstract}
Purpose. The aim of this study is to find the aspects that identify the self-organization cycle of beach handball and to compare these between genders.

Methods. Overall, 13 elite matches of each gender were analysed. We observed each team during the game, considering the following variables: offensive phase, ball possession, and attack outcome.

Results. A self-organization cycle was identified, characterized by a predominance of attack over defence. We found that ball possession changed constantly (> 84\%) and the same behaviour occurred in both genders. The difference between genders was that male teams were more likely to throw $(81 \%$ vs. $74 \%, p<0.001)$ and score a spectacular goal $(49 \%$ vs. $39 \%, p<0.001)$ when compared with female teams.

Conclusions. The self-organization cycle of beach handball involves a prevalence of attack over defence, with a higher probability of the offensive phase ending with a spectacular throw, and with a constant change of ball possession during the match (system attractors).
\end{abstract}

Key words: match analysis, internal logic, team sports

\section{Introduction}

Beach handball is an invasion team sport characterized by the opposition of specific actions between 2 teams in the same space, alternating among offensive, defensive, and transitions phases [1]. Some aspects are similar to indoor handball, but with specific characteristics and rules, such as the sand terrain, field dimensions, weather conditions [2], the scoring system by sets and a variety of throws that are specific [3]. As a result, these features could influence the action principles and action rules of the game [4], defining beach handball as a new sport.

In team sports, there are some functional parameters that interact with one another, such as rules, space, time, motor communication [4]. In beach handball, as previously mentioned, characteristics such as the scor- ing system and the weather conditions (humidity, temperature, and wind) may be important factors influencing the players' performance [2] and must be considered in the different game phases. The internal logic of the game (which includes rules and players' interactions) has been understood as a dynamic system [5-7] with a set of active and related elements that constitute a structure, with elements invariant in time, ensuring their own identity [8]. In this context, the attractors are the actions that allow to identify a pattern of organization of a system or team within the game [9].

As a system, the relationships between teammates and opponents can contribute to the modelling of the team sports organization. The cooperative and opposition relations within a complex and unpredictable context that the game constitutes require a great capacity of adaptation of the technical-tactical attitude

Correspondence address: Rafael Pombo Menezes, University of São Paulo, School of Physical Education and Sport of Ribeirão Preto, Ave Bandeirantes, 3900, Campus USP - 14040-907 - Ribeirão Preto-SP, Brazil, e-mail: rafaelpombo@usp.br

Received: June 17, 2020

Accepted for publication: February 8, 2021

Citation: Modolo F, dos Santos WR, Campos RRO, Morato MP, Menezes RP. Key features of beach handball self-organization: comparison between genders. Hum Mov. 2022;23(3):72-80; doi: https://doi.org/10.5114/hm.2022.107971. 
of the teams and players [4]. When teams develop new strategies, tactics, and techniques in order to solve the problems of game situations, such solutions generate results that influence the dynamics of the relationships among the elements and, thus, self-organize the system. The actions that do not occur so often in the game are called system fluctuations and represent a 'disturbance' in the system created spontaneously by the game through the relationships between players and teams [9].

Any team that wants to play a team sport will need to self-organize to accomplish the operational principles of attack, defence, and transition, in accordance with the different game rules, space, and score system of each team sport. Few studies are carried out with beach handball, and investigations on game analysis focus on anthropometric characteristics [2, 10, 11], motor actions, or technical-tactical actions executed in the offensive or defensive phase [12,13]. Cobos et al. [14] presented the game cycle of beach handball, considering the game cycle of handball (indoor). They highlighted the differences and the similarities of these 2 sports, explaining the characteristics of each game phase. Vázquez-Diz et al. [15] analysed the contextual factors and the decision-making that influenced the behaviour of finalization during the positional attack in beach handball, also exposing the differences between males and females. Dol et al. [3] showed the relation concerning types of throw between winning and losing female elite beach handball teams. They demonstrated that the winning teams performed a greater number of in-flight goals more effectively than the losing teams. Iannaccone et al. [16] investigated the efficiency of shots in beach handball (semifinal and final matches of senior, U18, and U12 teams), comparing male and female teams, and reported that no significant gender differences emerged for the different types of shots, shooting areas, or goal areas.

Although the contribution of previous studies is important for a better understanding of this sport, none of them analysed the self-organization in beach handball. Therefore, 2 main questions are pertinent for this study: (a) What are the aspects that define self-organization in beach handball? (b) Are these aspects different between genders? Thus, the aim of this study was to find the aspects that identify the self-organization cycle of beach handball and to compare these between genders.

\section{Material and methods}

\section{Sample}

Overall, 26 matches (13 men's and 13 women's matches; 1022 attacks of female teams and 1033 of male teams) of the $6^{\text {th }}$ World Handball Championship 2014 were analysed: 9 matches randomly drawn from the main round phase, in which the teams are classified in the group stage in a format of all against all; the 2 semifinal matches; and the finals (bronze and gold medals). The choice of the $6^{\text {th }}$ World Handball Championship was made because the researchers obtained the videos through a member of the technical staff of one team.

The selected matches represented the highest level of beach handball, ensuring control over opposition quality $[17,18]$. Only the major sets of each game were analysed. The shoot-out sets were not investigated because they have different characteristics than the major sets ( 1 vs. goalkeeper, not 4 vs. 3 ).

\section{Instruments}

The videos were observed with the Windows Media Player 10 software and the data were annotated in an ad hoc spreadsheet in Microsoft Office Excel 2010.

\section{Procedures}

The analysis protocol was developed by 3 researchers with previous research experience in sports science (FM: 5 years, WRS: 7 years, RROC: 7 years) and taking a master's course in sports science. The performance indicators [19] are presented in Table 1.

Furthermore, the performance indicators were revised by 2 more experienced researchers $(\mathrm{PhD})$. One of them (MPM) was a coach and game analyst for the Brazilian women's goalball team; the other one (RPM) was a game analyst for the Brazilian women's beach handball team (in a world championship). These 2 researchers also supervised the entire analysis protocol. The observational protocol elaborated by the 5 researchers is presented in Figure 1.

Data reliability was calculated by the same 3 researchers who developed the performance indicators. First, they went through a process of criteria standardization and agreement by consensus to minimize the observation bias [20]. Inter-observer reliability was verified with the analysis of 4 matches, which represented $15 \%$ of the sample. Intra-observer reliability 


\section{HUMAN MOVEMENT}

F. Modolo et al., Internal logic of beach handball

Table 1. Description of the performance variables

Offensive phase

Concluded: attacks finalized with a throw to the goal

Interrupted: attacks finalized without a throw to the goal

Ball possession

Kept: the attacking team kept ball possession at the end of the attack, keeping in the offensive phase

Changed: the attacking team scored or lost ball possession; in both situations, the team switched to the defensive phase

Attack outcome

Simple goal: 1 point scored by any team

Spectacular goal: 2 points scored by any team (in-flight goal, spin goal, specialist goal, direct goal, or 6-meter goal)

Goalkeeper's defence: throw saved by the opponent goalkeeper; when a block by the defenders occurred but the ball kept moving towards the goal with a goalkeeper's intervention, this was noted as goalkeeper's defence instead of block

Block: throw blocked by an opponent defender (except the goalkeeper) when the ball trajectory moved towards the goal

Goalpost: throw that hit the goalpost and did not score

Out: throwing out without touching any player

Interception: interruption of the ball trajectory after a pass, or when the defender/goalkeeper anticipated a forward pass from the attacking team

Attack violation: interruption of the offensive phase originated by a foul or violation of the rules by any player of the attacking team

Defence violation: interruption of the opposition team attack caused by a foul or violation of the rules

by the defending team

Attack error: loss of ball possession by the attacking team without involving the criteria of interception, attack violation, or defence violation; examples: wrong passes or wrong receptions

Referee intervention: fair play moments or a referee intervention that interrupted the match

\begin{tabular}{|c|c|c|c|c|c|c|}
\hline \hline SET & TEAM & VIDEO TIME & OFFENSIVE PHASE & ATTACK OUTCOME & BALL POSSESSION & MATCH-SCORE \\
\hline $1^{\circ}$ & TEAM A & $09: 15$ & CF & G2 & T & $2 \times 0$ \\
\hline $1^{\circ}$ & TEAM B & $09: 34$ & CF & G2 & T & $2 \times 2$ \\
\hline $1^{\circ}$ & TEAM A & $09: 51$ & CF & DG & M & $2 \times 2$ \\
\hline $1^{\circ}$ & TEAM A & $10: 06$ & CF & G1 & T & $3 \times 2$ \\
\hline $1^{\circ}$ & TEAM B & $10: 25$ & SF & FV & M & $3 \times 2$ \\
\hline $1^{\circ}$ & TEAM B & $10: 54$ & CF & G2 & T & $3 \times 4$ \\
\hline $1^{\circ}$ & TEAM A & $11: 11$ & CF & G2 & T & $5 \times 4$ \\
\hline $1^{\circ}$ & TEAM B & $11: 30$ & SF & FV & T & $5 \times 4$ \\
\hline $1^{\circ}$ & TEAM A & $11: 42$ & SF & FV & M & $5 \times 4$ \\
\hline $1^{\circ}$ & TEAM A & $12: 09$ & CF & G2 & T & $7 \times 4$ \\
\hline $1^{\circ}$ & TEAM B & $12: 25$ & SF & FV & M & $7 \times 4$ \\
\hline $1^{\circ}$ & TEAM B & $12: 37$ & CF & G2 & T & $7 \times 6$ \\
\hline $1^{\circ}$ & TEAM A & $12: 55$ & CF & G2 & T & $9 \times 6$ \\
\hline $1^{\circ}$ & TEAM B & $13: 14$ & CF & G2 & T & $9 \times 8$ \\
\hline $1^{\circ}$ & TEAM A & $13: 37$ & CF & G2 & T & $11 \times 8$ \\
\hline $1^{\circ}$ & TEAM B & $13: 52$ & CF & DG & M & $11 \times 8$ \\
\hline
\end{tabular}

Figure 1. Example of the observational protocol (ad hoc spreadsheet)

was based on 2 randomly selected matches $(7.7 \%$ of the sample). The intra- and inter-observer reliability were tested by Cohen's kappa [21-23] (Table 2). The second observation of the matches started a minimum of 15 days after the end of the first one.
Table 2. Reliability (Cohen’s kappa) for each variable

\begin{tabular}{lcc}
\hline Variables & Inter-observer & Intra-observer \\
\hline Offensive phase & 0.95 & 1.00 \\
Ball possession & 1.00 & 1.00 \\
Attack outcome & 0.88 & 0.96 \\
\hline
\end{tabular}




\section{Statistical analysis}

First, we counted the frequency of occurrence of all variables and then we calculated the percentage of occurrence of each variable for each match. Then, a series of $t$-tests were used to compare the data [24], with the match as a unit of analysis. For the intra-gender analysis (attacks concluded vs. interrupted and ball possession kept vs. changed), the paired samples $t$-test was used. For all inter-gender analyses, we ran independent sample tests. The Wilcoxon signed rank test and the Mann-Whitney $U$ test were, respectively, incorporated when the Shapiro-Wilk test revealed cases of non-normal distribution or the data failed Levene's test [25]. Statistical significance of the results was set at $p<0.05$ and the mean percentage of the 13 matches for each gender is presented in the results.

\section{Ethical approval}

The research related to human use has complied with all the relevant national regulations and institutional policies, has followed the tenets of the Declaration of Helsinki, and has been approved by the Research Ethics Committee of the University of São Paulo, School of Physical Education and Sport of Ribeirão Preto (CAAE: 67387017.7.0000.5659).

\section{Informed consent}

The referred Research Ethics Committee waived the presentation of the informed consent.

\section{Results}

The analysis of the offensive phase of the teams participating in the $6^{\text {th }}$ World Handball Championship revealed that teams of both genders tended to conclude their attacks with a throw (females: $73.9 \pm 4.0 \%, \mathrm{t}_{12}=$ 21.6, $p<0.001$; males: $80.6 \pm 3.5 \%, \mathrm{t}_{12}=31.4, p<0.001$ ).
However, male teams were more likely to throw when compared with women $\left(\mathrm{t}_{24}=-4.55, p<0.001\right)$.

We also observed that ball possession was changed after the majority of attacks concluded and interrupted, regardless of gender (Table 3). In the comparison between genders, there were more ball possession changes in the concluded attacks in men's matches $(U=39.0, z=-2.33, p=0.020)$ and in the interrupted attacks in women's matches $\left(\mathrm{t}_{24}=4.50, p<0.001\right)$. There was no difference in maintaining ball possession, either for concluded $\left(\mathrm{t}_{24}=-1.78, p=0.088\right)$ or for interrupted attacks $\left(\mathrm{t}_{24}=0.36, p=0.721\right)$.

The spectacular goal was the attack outcome most frequent in both genders (Figure 2). When the genders were compared, men had a higher percentage of spectacular goals $\left(\mathrm{t}_{24}=-3.62, p=0.001\right)$, while women had a higher percentage of blocks $(U=38.0, z=-2.39$, $p=0.017)$ and attack errors $(U=28.0, z=-2.90, p=$ $0.004)$.

\section{Discussion}

This study aimed to identify the self-organization cycle of high-level beach handball and to verify if there were differences in this respect between female and male matches. A piece of evidence presented in beach handball was the occurrence of throws to the goal, pointed out as one of the main attractors of self-organization in this sport for both genders. This may be related to the specific characteristics of the sport, which presents offensive numerical superiority guaranteed throughout the match by the rules themselves (except when one team has a punished player) [14]. According to these rules, the team with the ball could replace the goalkeeper to attack with 4 attackers against 3 defenders, which increases the chances to conclude the offensive phase with a throw.

Table 3. Ball possession at the end of the attack in female and male elite beach handball

\begin{tabular}{|c|c|c|c|c|c|}
\hline \multirow{2}{*}{ Variables } & \multicolumn{2}{|c|}{ Ball possession } & \multirow{2}{*}{$t$-test } & \multirow{2}{*}{$\mathrm{df}$} & \multirow{2}{*}{$p$} \\
\hline & Kept & Changed & & & \\
\hline \multicolumn{6}{|c|}{ Attacks concluded } \\
\hline Women & $5.8 \pm 2.2 \%$ & $68.1 \pm 5.4 \%$ & -41.5 & 12 & $<0.001$ \\
\hline Men & $7.7 \pm 3.1 \%$ & $72.8 \pm 4.0 \%$ & -24.5 & 12 & $<0.001$ \\
\hline \multicolumn{6}{|c|}{ Attacks interrupted } \\
\hline Women & $8.6 \pm 2.7 \%$ & $17.5 \pm 3.6 \%$ & 10.1 & 12 & $<0.001$ \\
\hline Men & $8.2 \pm 2.9 \%$ & $11.2 \pm 3.6 \%$ & $z=-1.87 *$ & - & 0.062 \\
\hline
\end{tabular}

* Wilcoxon signed rank test 


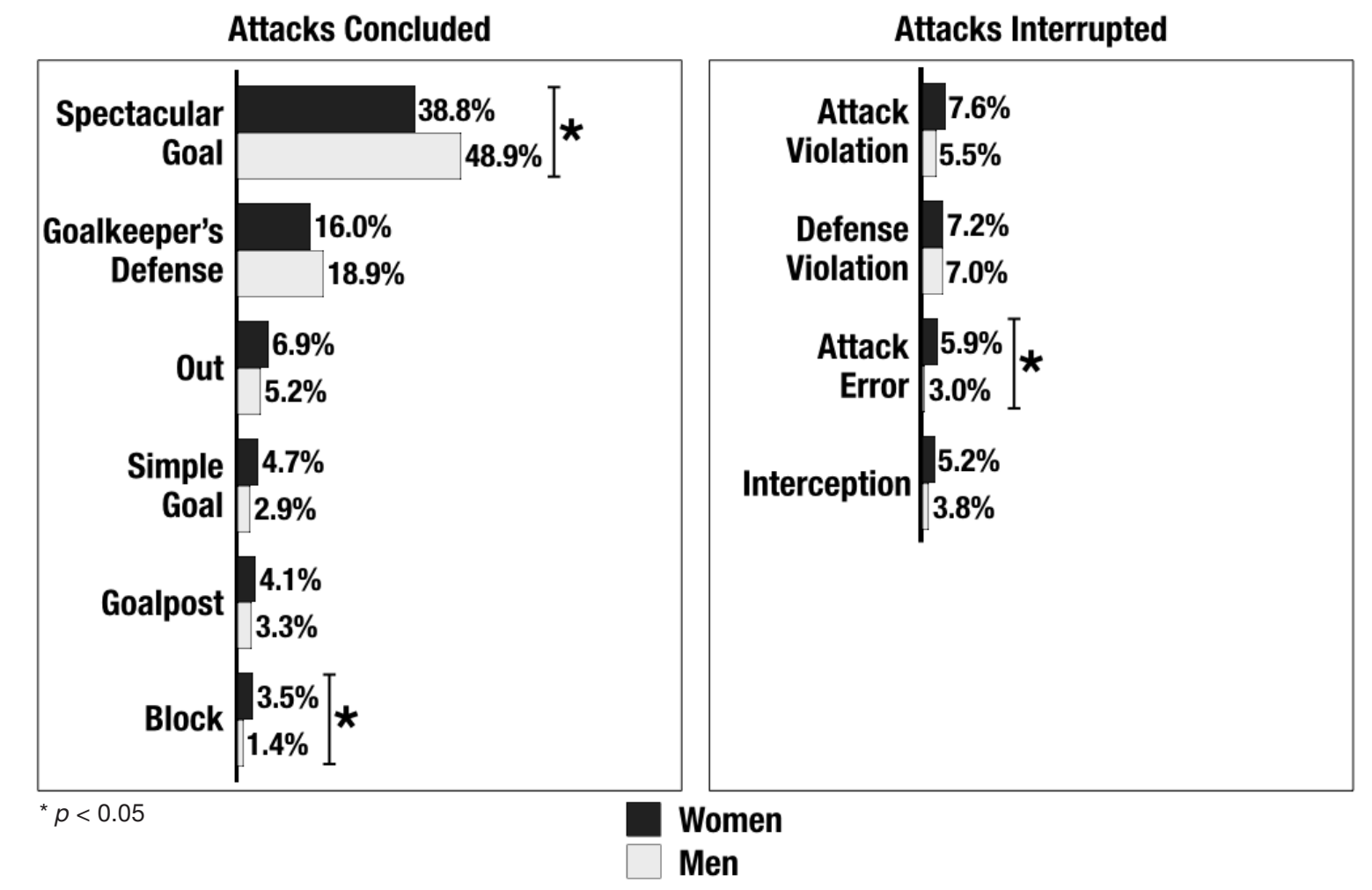

Figure 2. Attack outcome after attacks concluded or interrupted in beach handball

Considering that the majority of the attacks will end with a throw to the goal, another finding of this research is that the spectacular goal, characterized by difficult execution and worth 2 points, was the most frequent attack outcome observed in both genders, as presented by other authors [15]. In relation to the previous feature, it seems that men had more chances to score than women in beach handball. Given that there was a greater frequency of spin shots for both genders in the 2014 competition [26, 27], and also in European youth teams [12] and in a specific tournament [16], although the spectacular goal can be performed in different ways (e.g. $360^{\circ}$ spin shot, in-flight shot, penalty shot, shot by a goalkeeper or specialist player) [28], the fact that men were more effective may be explained by differences in strength of lower and upper limbs between genders or by the level of training [29]. We also identified that men were more likely to score a spectacular goal, while women executed more blocks and attack errors [15], which explains the observed percentage differences.

On the other hand, the male and female gold medal teams in the $6^{\text {th }}$ World Handball Championship 2014 presented a greater frequency and effectiveness in in-flight throws compared with the other teams [26, 27]. Considered a more complex technical-tactical aspect owing to the involvement of 2 players of the same team [30], the performance of the in-flight throw can be an indicator of the level of beach handball teams, especially female ones [3] - but, as previously highlighted, it seems that the same is true for male teams [27].

The constant change of ball possession between teams was identified as another attractor for the selforganization cycle of beach handball in both genders, with reference to both completed and interrupted attacks. This can be explained by the fact that completed attacks are considered to be those in which there is a shot towards the target, from which different results can occur (as shown in Figure 2: goal, shooting out, goalkeeper's defence, block, and goalpost). Thus, there were large percentages of changing ball possession, even in concluded attacks (see Table 3). In interrupted attacks, in which there is a loss of ball possession without shoot, the same behaviour of changing ball possession was observed (see Table 3) - through attack error, interception, and defensive or offensive violations.

From this perspective, there is no reason for the team in the offensive phase to accelerate their attack (except when the team is losing in the final moments of a match), since ball possession is unlikely to be maintained with the conclusion or interruption of the attack. It seems more interesting for the attacking team to wait for the substitution of the goalkeeper to attack in numerical superiority [14] and apply offensive actions that allow the accomplishment of the spectacular goal. This attractor becomes even more evident if 
one considers the observed small percentage of direct goals (goalkeeper's throws) performed by male $(<1.8 \%)$ and female $(<1.1 \%)$ teams in the 2014 competition [31].

After ball possession change, the team that loses the ball does not necessarily need to press the opponent in the offensive court as this team will have numerical inferiority, which guarantees advantage to the opponent's attack. It is more advantageous for the defence to return to its defensive field and rapidly substitute the specialist player with the goalkeeper, to try to induce the opponent's attack with a simple goal attempt, since it is unlikely that it will be able to prevent the execution of the throw. This guaranteed numerical superiority of the attack over the defence could be a possible indicator to the coaches not to prioritize the occurrence of counter-attacks in beach handball. Unlike in the transition phases of indoor handball, in which a quick transition can provide a numerical advantage attack [32], in beach handball, owing to the numerical advantage guaranteed [14], there is no need for the attacking team to look for a quick offensive transition or even try a direct goal from the goalkeeper. It does not mean that these strategies cannot be applied; however, they present a higher risk of failure and their use could be reserved for critical moments (e.g. matches tied in the final minutes or when a team needs to de- crease big advantages in the score). The self-organization cycle of high-level beach handball is represented in Figure 3.

From the offensive point of view, when there is a throw to the goal followed by recovery of ball possession by the same team that threw, there is also a fluctuation of the system. This can occur mainly owing to the action of the goalkeeper, who can prevent the goal (spectacular or simple) but does not recover the ball, giving the opponents a new opportunity to attack. Another fluctuation highlighted is related to the attempt of a simple goal in a deliberate manner, depending on the match status and time. These fluctuations happened when the score was very close and with little time remaining in the set. The team holding match score advantage and the last ball possession of the set prefers to choose a simple goal throw, which presents less complexity (and therefore less risk of error), but which is enough to win the set or the match (maintaining, for example, 2 ball possessions of advantage).

The influence of match status is not exclusive to beach handball. In football, the best European teams showed a shorter sequence of passes when they were winning the matches [33] and the attackers were more active when their team led the match, while defenders had a higher work rate during periods in which

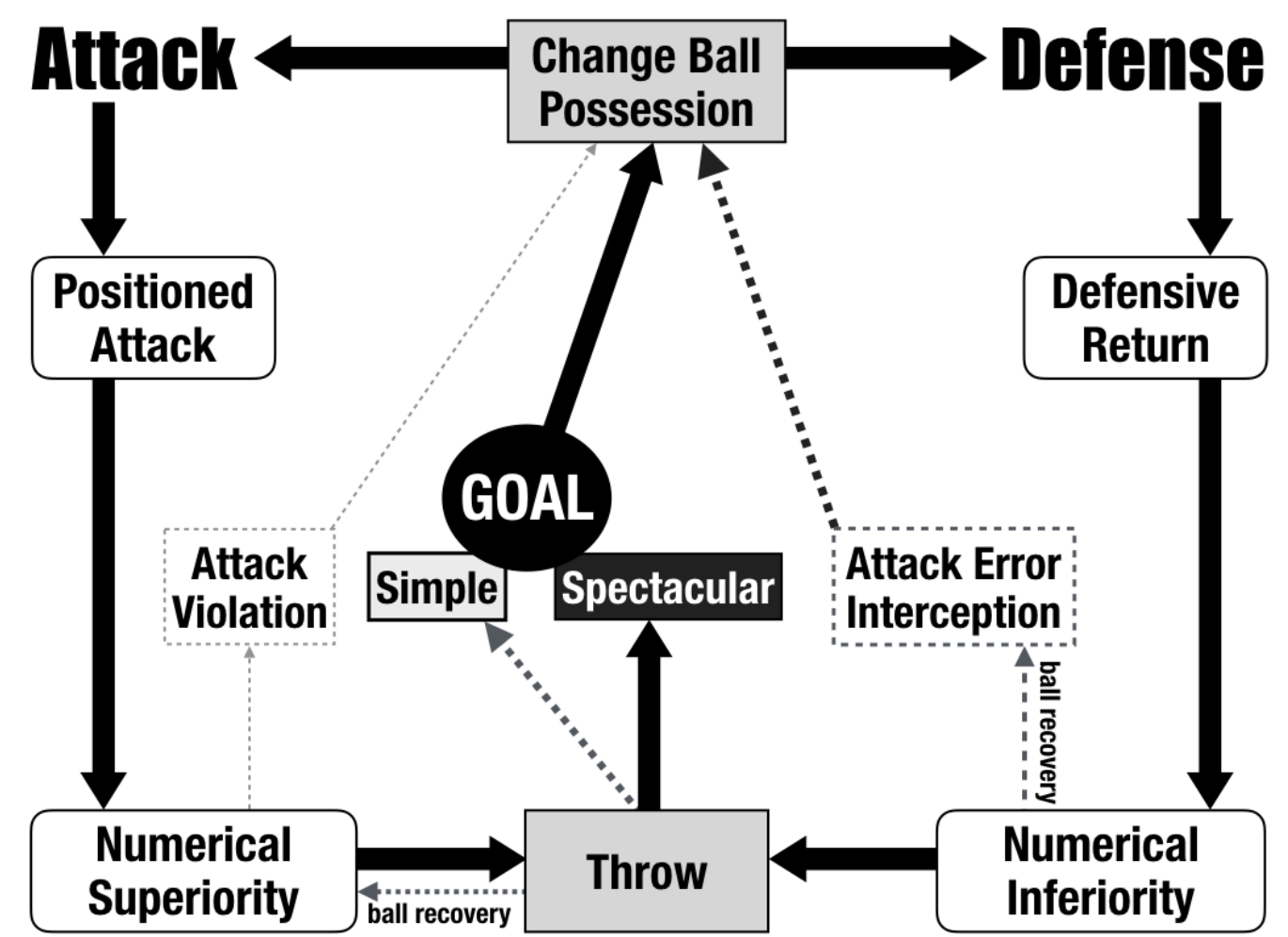

Figure 3. Self-organization cycle of high-level beach handball. Full and dark arrows indicate the system attractors (events most likely to occur). Dashed arrows denote system fluctuations (the lower the thickness and the colour intensity of the arrow, the lower the probability of occurrence) 
the defending team were losing or the score was tied [34]. Considering the match status and time, in the last quarter of close NBA games, the defenders tend to perform more fouls to stop the game and reduce the chances to score points per possession by the fouling team via free-throws [35].

In addition, this strategy of opting for the simple goal used by the teams that are winning is supported by beach handball official rules. As the points are not accumulated for the next set, it is necessary to win the first 2 sets, regardless of the general score, to win a match. If there is a tie of one set for each team, the game will be decided in the third set (shoot-out system). A total of 5 alternate throws (attacker vs. goalkeeper) are performed by each team, with the team that scores the most points at the end being the winners [28]. As in volleyball, each set can be considered a new match since the teams will start the next set with zero points. In this way, player behaviour will be influenced by match status in each set. The players can either try harder to win the set or save themselves physically to return more recovered to the next set, when it is impossible to overturn the opposing team's advantage. A similar effect was evidenced in volleyball, with the demonstration that teams made different decisions depending on the score in each set. In sets with nontied scores, teams took more risky decisions, while in sets with tied scores, teams opted for safer tactical strategies [17].

\section{Conclusions}

Considering the guiding questions of this study, the self-organization cycle of beach handball is defined as a game with prevalence of attack over defence, which helps to identify patterns of play in this sport and to understand its dynamics. This contributes to the increase of knowledge about the game among scientists, players, and coaches.

Beach handball presents a higher probability of the offensive phase ending with a spectacular throw and with a constant change of ball possession during the match (system attractors). The fluctuation of the system occurs when the defending team recovers the ball before the opponent's throw to the goal (turnovers), when the execution of a spectacular goal is difficult, inducing the achievement of only a simple goal, or when the attacking team keeps the ball after a throw to the goal or scores a simple goal purposely (with a very close score). In addition, the cycle involved the same behaviour for both genders, although men presented more chances to score than women.
On the basis of the understanding of the self-organizational cycle in beach handball, this study presents the main characteristics of each phase of the game, as well as the indicators that may guide the creation of specific game models, associating the specific demands with the coaches' context (i.e. competitive level, player characteristics). Thus, the findings of this study may assist the coach in their professional performance, as the knowledge of the cycle and its main attractors can influence training session planning through prioritizing exercises that will develop the characteristics of each phase of the game (offensive/defensive/transitions).

Indeed, the coach must design the guidelines for their team's game model from the characteristics of the self-organization cycle of beach handball, respecting its attractors, since interactions between players from different teams can produce system fluctuations and unbalance the match result (e.g. recovering ball possession without the opponent's throws to the goal). Coaches can therefore plan strategies to provoke opponents' turnovers and use fluctuations in favour of their own team (in the defensive phase), as well as reinforce strategies for scoring spectacular goals (in the offensive phase).

The main limitations of this study are related to the analysis of the shoot out (as an exceptional game situation, played when each team wins a set), which has different dynamics than the other sets. In addition, future studies can bring analyses of the match status, the position of the team on the court (whether it plays for or against the sun), and different stages of the competition (group stage or playoffs).

\section{Disclosure statement}

No author has any financial interest or received any financial benefit from this research.

\section{Conflict of interest}

The authors state no conflict of interest.

\section{References}

1. Menezes RP, Marques RFR, Nunomura M. Early sport specialization and the teaching of invasion games [in Portuguese]. Movimento. 2014;20(1):351-373; doi: 10.22456/1982-8918.40200.

2. Jimenez-Olmedo JM, Penichet-Tomas A, Becerra MO, Pueo B, Espina-Agullo JJ. Relationships between anthropometric parameters and overarm throw in elite beach handball. Hum Mov. 2019;20(2):16-24; doi: 10.5114/hm.2019.79394.

3. Dol G, Onetto V, Carbonell V, González-Ramírez A. Analysis of throwing performance in elite women's beach handball [in Catalan]. Apunts Educ Fis Deportes. 
2020;141:49-54; doi: 10.5672/apunts.2014-0983.es. (2020/3).141.06.

4. Gréhaigne J-F, Godbout P. Tactical knowledge in team sports from a constructivist and cognitivist perspective. Quest. 1995;47(4):490-505; doi: 10.1080/003362 97.1995.10484171.

5. McGarry T, Anderson DI, Wallace SA, Hughes MD, Franks IM. Sport competition as a dynamical self-organizing system. J Sports Sci. 2002;20(10):771-781; doi: 10.1080/026404102320675620.

6. Passos P, Araújo D, Davids K. Self-organization processes in field-invasion team sports: implications for leadership. Sports Med. 2013;43(1):1-7; doi: 10.1007/ s40279-012-0001-1.

7. Reed D, Hughes MD. An exploration of team sport as a dynamical system. Int J Perform Anal Sport. 2006; 6(2):114-125;doi:10.1080/24748668.2006.11868377.

8. D’Ottaviano IML, Bresciani Filho E. Self-organization and creation [in Portuguese]. MultiCiência. 2004;3(1): $1-23$.

9. Morato MP, Pimentel Gomes MS, Gavião de Almeida JJ. The self-organizations processes of goalball [in Portuguese]. Rev Bras Cienc Esporte. 2012;34(3):741-760; doi: 10.1590/S0101-32892012000300015.

10. Pueo B, Jimenez-Olmedo JM, Penichet-Tomas A, Becerra MO, Espina Agullo JJ. Analysis of time-motion and heart rate in elite male and female beach handball. J Sports Sci Med. 2017;16(4):450-458.

11. Sena JEA, Gomes ALM, Mimbacas A, Ferreira UMG. Dermatoglyph, somatotype and body composition in beach handball: comparative study among different level of sportive qualification [in Portuguese]. Motricidade. 2012;8(2):567-576.

12. Morillo-Baro JP, Reigal RE, Hernández-Mendo A. Analysis of positional attack in beach handball male and female with polar coordinates [in Spanish]. Rev Int Cienc Deporte. 2015;11(41):226-244; doi: 10.5232/ricyde 2015.04103.

13. Gkagkanas K, Hatzimanouil D, Skandalis V, Dimitriou S, Papadopoulou SD. Defense tactics in high-level teams in beach handball. J Phys Educ Sport. 2018; 18(2):914-920; doi: 10.7752/jpes.2018.02135.

14. Cobos DL, Sánchez Sáez JA, Morillo Baro JP, Sánchez Malia JM. Beach handball game cycle [in Spanish]. Rev Int Deportes Colect. 2018;34:89-100.

15. Vázquez-Diz JA, Morillo-Baro JP, Reigal RE, MoralesSánchez V, Hernández-Mendo A. Contextual factors and decision-making in the behavior of finalization in the positional attack in beach handball: differences by gender through polar coordinates analysis. Front Psychol. 2019;10:1386; doi: 10.3389/fpsyg.2019.01386.

16. Iannaccone A, Fusco A, Conte D, Cortis C. Notational analysis of beach handball. Hum Mov. 2022;23(1); doi: 10.5114/hm.2021.101757.

17. Marcelino R, Mesquita I, Sampaio J. Effects of quality of opposition and match status on technical and tactical performances in elite volleyball. J Sports Sci. 2011; 29(7):733-741; doi: 10.1080/02640414.2011.552516.

18. O’Donoghue P, Holmes L, Robinson G. Doing a research project in sport performance analysis. Abingdon: Routledge; 2017.

19. Hughes MD, Bartlett RM. The use of performance indicators in performance analysis. J Sports Sci. 2002; 20(10):739-754; doi: 10.1080/026404102320675602.

20. Anguera MT, Mendo AH. Observational methodology in sport sciences [in Spanish]. E-balonmano.com: Rev Cienc Deporte. 2013;9(3):135-160.

21. Thomas JR, Nelson JK, Silverman SJ. Research methods in physical activity, $7^{\text {th }}$ ed. Champaign: Human Kinetics; 2015.

22. James N, Taylor J, Stanley S. Reliability procedures for categorical data in performance analysis. Int J Perform Anal Sport. 2007;7(1):1-11; doi: 10.1080/24748668. 2007.11868382.

23. Sainani KL. Reliability statistics. PM R. 2017;9(6): 622-628; doi: 10.1016/j.pmrj.2017.05.001.

24. Lynch P, Carroll R. To compare the type of passing in Gaelic football at senior inter-county level historically to modern day. Int J Perform Anal Sport. 2017;17(6):986995; doi: 10.1080/24748668.2017.1416526.

25. O’Donoghue P, Holmes L. Data analysis in sport. Abingdon: Routledge; 2014.

26. International Handball Federation. Overall team statistics of VI Men's Beach Handball Championship. Available from: https://archive.ihf.info/en-us/ihfcompetitions/beachhandball/vibeachhandballworldchampionships-men/statistics.aspx.

27. International Handball Federation. Overall team statistics of VI Women's Beach Handball Championship. Available from: https://archive.ihf.info/en-us/ihfcompetitions/beachhandball/vibeachhandballworldchampionships-women/statistics.aspx.

28. International Handball Federation. IX. Rules of the game. b) Beach Handball. 2021. Available from: https://www.ihf.info/sites/default/files/202110/09B\%20-\%20Rules\%20of\%20the\%20Game_ Beach\%20Handball_E_0.pdf.

29. Granados C, Izquierdo M, Ibañez J, Bonnabau H, Gorostiaga EM. Differences in physical fitness and throwing velocity among elite and amateur female handball players. Int J Sports Med. 2007;28(10):860-867; doi: 10.1055/s-2007-964989.

30. Da Silva KP, Menezes RP. The offensive game of beach handball: structure and technical-tactical aspects of positional attack [in Portuguese]. Cuad Psicol Deporte. 2018;18(3):209-221.

31. International Handball Federation. Beach Handball World Championship in Brazil. Overall team statistics: tournament summary as of 2014-07-27. Available from: http://archive.ihf.info/files/competitiondata/32b467b5c7c8-48bc-aee6-fd541a545ca3/pdf/TOPTeam.pdf.

32. Menezes RP, Morato MP, Marques RFR. Offensive and defensive strategies in handball during the transition 


\section{HUMAN MOVEMENT}

F. Modolo et al., Internal logic of beach handball

phase from the perspective of experienced coaches [in Portuguese]. J Phys Educ. 2016;27(1):e2753; doi: 10.4025/ jphyseduc.v27i1.2753.

33. Paixão P, Sampaio J, Almeida CH, Duarte R. How does match status affects the passing sequences of top-level European soccer teams? Int J Perform Anal Sport. 2015;15(1):229-240; doi: 10.1080/24748668.2015.11 868789 .

34. Redwood-Brown A, O’Donoghue P, Robinson G, Neilson $P$. The effect of score-line on work-rate in English FA Premier League soccer. Int J Perform Anal Sport. 2012; 12(2):258-271; doi: 10.1080/24748668.2012.11868598.

35. Gomez MA, Gasperi L, Lupo C. Performance analysis of game dynamics during the $4^{\text {th }}$ game quarter of NBA close games. Int J Perform Anal Sport. 2016;16(1):249263; doi: 10.1080/24748668.2016.11868884. 